\title{
LARGE-SCALE STOCHASTIC HEREDITARY SYSTEMS UNDER MARKOVIAN STRUCTURAL PERTURBATIONS. PART III. QUALITATIVE ANALYSIS
}

\author{
G. S. LADDE \\ Received 14 January 2005; Revised 26 October 2005; Accepted 26 October 2005
}

In this final part of the work, the convergence and stability analysis of large-scale stochastic hereditary systems under random structural perturbations is investigated. This is achieved through the development and the utilization of comparison theorems in the context of vector Lyapunov-like functions and decomposition-aggregation method. The byproduct of the investigation suggests that the qualitative properties of decoupled stochastic hereditary subsystems under random structural perturbations are preserved, as long as the self-inhibitory effects of subsystems are larger than cross-interaction effects of the subsystems. Again, it is shown that these properties are affected by hereditary and random structural perturbations effects. It is further shown that the mathematical conditions are algebraically simple, and are robust to the parametric changes. Moreover, the work generates a concept of block quasimonotone nondecreasing property that is useful for the investigation of hierarchic systems. These results are further extended to the integrodifferential equations of Fredholm type.

Copyright (c) 2006 G. S. Ladde. This is an open access article distributed under the Creative Commons Attribution License, which permits unrestricted use, distribution, and reproduction in any medium, provided the original work is properly cited.

\section{Introduction}

A variety of problems that arise in the fields of engineering, medical, social, and physical sciences under hereditary and random environmental perturbations can be modeled by a system of stochastic functional differential equations. A feasible model for such a system is an Itô-type stochastic functional differential system perturbed by a finite-state Markov process. In this work, by using decomposition-aggregation method $[2,3,6,11,9,12-$ $14,18]$, we propose to study the qualitative behavior of a solution of such a large-scale stochastic hereditary system. Moreover, by utilizing variational comparison theorems [9] for each isolated subsystem in the context of energy/Lyapunov-like functions and the nature of interactions among the subsystems of a large-scale system, a very general variational comparison theorem is formulated. These comparison theorems are used to investigate

Hindawi Publishing Corporation

Journal of Applied Mathematics and Stochastic Analysis

Volume 2006, Article ID 24643, Pages 1-10

DOI 10.1155/JAMSA/2006/24643 
various modes of convergence of a solution process of the stochastic large-scale hereditary system. Stability results for the trivial solution of the stochastic large-scale system are also obtained.

In Section 2, variational comparison results of isolated subsystems (DHS) [9] are extended to large-scale stochastic hereditary systems under Markovian structural perturbations (LHS). The comparison results of this section are utilized to investigate the convergence and stability analysis of hierarchic systems in Section 3. The byproduct of the study generates a concept of block quasimonotone nondecreasing property [1,2, 15-17]. Moreover, the scope and the significance of the presented results are outlined in Section 4 . In particular, it contains remarks concerning the effects of the hereditary and random perturbations of the system on the convergence and stability. The presented results extend and generalize the earlier work $[1-8,13-18]$ in a systematic and unified way.

\section{Variational comparison theorems}

Let us recall large-scale stochastic hereditary system (LHS) described in [9]. It is decomposed into smaller, simpler, and suitable $p$-interconnected subsystems (perturbed) of the following form:

$$
d x^{i}=\left[\mathbf{a}^{i}\left(t, x_{t}^{i}, \eta(t)\right)+\mathbf{c}^{i}\left(t, x_{t}, \eta(t)\right)\right] \cdot d \boldsymbol{\xi}(t), \quad x_{t_{0}}^{i}=\varphi_{0}^{i}
$$

where, $\mathbf{a}^{i}=\left(a_{0}^{i T}, a_{1}^{i T}, \ldots, a_{j}^{i T}, \ldots, a_{m}^{i T}\right)^{T}$ and $\mathbf{c}^{i}=\left(c_{0}^{i T}, c_{1}^{i T}, \ldots, c_{j}^{i T}, \ldots, c_{m}^{i T}\right)^{T}, a_{j}^{i} \in C\left[J \times C^{n_{i}} \times\right.$ $\left.R, R^{n_{i}}\right]$ and $c_{j}^{i} \in C\left[J \times C^{n} \times R, R^{n_{i}}\right]$ for $j \in I(0, m) ; \varphi_{0}=\left(\left(\varphi_{0}^{1}\right)^{T},\left(\varphi_{0}^{2}\right)^{T}, \ldots,\left(\varphi_{0}^{p}\right)^{T}\right)^{T}, x=$ $\left(\left(x^{1}\right)^{T},\left(x^{2}\right)^{T}, \ldots,\left(x^{p}\right)^{T}\right)^{T}$, and $\sum_{i=1}^{p} n_{i}=n$ for each $i \in I(1, p)$; the interactions (perturbations) among the $p$ subsystems of system (LHS) are described by $c^{i}$.

In this section, analogous to the results of [9, Section 3], we develop a few auxiliary comparison results for stochastic large-scale hereditary system (LHS). For this purpose, we utilize an energy/Lyapunov-like function associated with each decoupled/isolated stochastic hereditary subsystem (DHS) corresponding to (LHS) under Markovian structural perturbations:

$$
d x^{i}=\mathbf{a}^{i}\left(t, x_{t}^{i}, \eta(t)\right) \cdot d \boldsymbol{\xi}(t), \quad x_{t_{0}}^{i}=\varphi_{0}^{i}
$$

in $[10]$ for $i \in I(1, p)$.

We use the same auxiliary systems of differential equations as in (DAS) as described in [9]. As stated before, we use vector Lyapunov-like functions associated with each isolated system $i \in I(1, p)$ in (DHS). By following the definition in [9], we define $D_{(\mathrm{LHS})}^{+} V^{i}(s, \varphi$, $\left.z^{i}\left(t, s, \varphi^{i}(0)\right), \eta(s)\right)$, for each subsystem of large-scale system (LHS).

Remark 2.1. With respect to each subsystem $i \in I(1, p)$ of large-scale system (LHS), an operator similar to operator $L_{i}^{D}$ defined in [9] with regard to isolated subsystems can also be defined, analogously, and it is denoted by $L_{i}^{C}$. For each $i \in I(1, p)$, we have

$$
D_{\mathbf{F}_{i}}^{+} V^{i}\left(s, \varphi, z^{i}\left(t, s, \varphi^{i}(0)\right), \eta(s)\right) \leq L_{i}^{C} V^{i}\left(s, \varphi, z^{i}\left(t, s, \varphi^{i}(0)\right), \eta(s)\right)
$$


where $L_{i}^{C}$ is defined and can be represented as

$$
\begin{aligned}
& L_{i}^{C} V^{i}\left(s, \varphi, z^{i}\left(t, s, \varphi^{i}(0)\right), \eta(s)\right) \\
& =L_{i}^{D} V^{i}\left(s, \varphi^{i}, z^{i}\left(t, s, \varphi^{i}(0)\right), \eta(s)\right)+I^{i}\left(\mathbf{c}^{i}(t, s, \varphi, \eta(s))\right) \\
& I^{i}\left(\mathbf{c}^{i}(t, s, \varphi, \eta(s))\right) \\
& =\frac{1}{2} \operatorname{tr}\left(\frac{\partial^{2}}{\partial z^{i 2}} V^{i}\left(s, \varphi^{i}, z^{i}\left(t, s, \varphi^{i}(0)\right), \eta(s)\right) \Theta_{i}^{C}\left(t, s, \varphi^{i}(0)\right), \eta(s)\right) \\
& \quad+\frac{\partial}{\partial z^{i}} V^{i}\left(s, z^{i}\left(t, s, \varphi^{i}(0)\right), \eta(s)\right)\left[\frac{1}{2} \operatorname{tr}\left(\frac{\partial^{2}}{\partial x^{i} \partial x^{i}} z_{i}\left(t, s, \varphi^{i}(0)\right) \Lambda_{i}^{C}(s, \varphi, \eta(s))\right)\right. \\
& \left.\quad+\Phi^{i}\left(t, s, \varphi^{i}(0)\right) M_{i}^{C}(s, \varphi, \eta(s))\right], \\
& M_{i}^{C}(s, \varphi, \eta(s))=\lim _{h \rightarrow 0^{+}} \frac{1}{h} E\left[\mathbf{c}^{i}(s, \varphi, \eta(s)) \cdot \Delta \xi(s) \mid F_{s}\right], \\
& \Lambda_{i}^{C}\left(s, \varphi^{i}, \varphi, \eta(s)\right) \\
& =\lim _{h \rightarrow 0^{+}} \frac{1}{h} E\left[\left(\left[\mathbf{a}^{i}\left(s, \varphi^{i}, \eta(s)\right)+\mathbf{c}^{i}(s, \varphi, \eta(s))\right] \cdot \Delta \xi(s)\right)\right. \\
& \left.\quad \times\left(\left[\mathbf{a}^{i}\left(s, \varphi^{i}, \eta(s)\right)+\mathbf{c}^{i}(s, \varphi, \eta(s))\right] \cdot \Delta \xi(s)\right)^{T} \mid F_{s}\right]-\Lambda_{i}^{D}\left(s, \varphi^{i}, \eta(s)\right), \\
& \Theta_{i}^{C}\left(t, s, \varphi^{i}(0), \varphi, \eta(s)\right)=\Phi^{i}\left(t, s, \varphi^{i}(0)\right) \Lambda_{i}^{C}\left(s, \varphi^{i}, \varphi, \eta(s)\right) \Phi^{i T}\left(t, s, \varphi^{i}(0)\right) .
\end{aligned}
$$

Before we present a main variational comparison result for large-scale system, we present two lemmas. The first lemma is analogous to [9, Lemma 3.1] with respect to large-scale system (LHS). The second lemma is an extension of the well-known maximal solution for systems of functional differential equations [15-17].

Lemma 2.2. Assume that all the hypotheses of [9, Lemma 3.1] are satisfied. Then,

$$
\begin{aligned}
D_{(L H S)}^{+} & V^{i}\left(s, \varphi, z^{i}\left(t, s, \varphi^{i}(0)\right), \eta(s)\right) \\
\quad= & L_{i}^{C} V^{i}\left(s, \varphi, z^{i}\left(t, s, \varphi^{i}(0)\right), \eta(s)\right)+D_{\eta}^{+} V\left(s, z^{i}\left(t, s, \varphi^{i}(0)\right), \eta(s)\right),
\end{aligned}
$$

where $(t, \varphi) \in R_{+} \times \mathscr{C}^{n} ; D_{(L H S)}^{+} V^{i}\left(s, \varphi, z^{i}\left(t, s, \varphi^{i}(0)\right), \eta(s)\right), D_{\eta}^{+} V^{i}\left(s, z^{i}\left(t, s, \varphi^{i}(0)\right), \eta(s)\right)$, and $L_{i}^{C} V^{i}\left(s, \varphi, z^{i}\left(t, s, \varphi^{i}(0)\right), \eta(s)\right)$ are as defined above.

Prior to stating the second lemma, we need to introduce a concept of block quasimonotone nondecreasing property of a comparison function.

Definition 2.3. For each $i \in I(1, p)$ and $j \in I(1, q)$, Denote $u^{i j} \in R_{+}^{q_{i}}$, and $u(j)$ by $u(j)=$ $\left(\left(u^{1 j}\right)^{T},\left(u^{2 j}\right)^{T}, \ldots,\left(u^{i j}\right)^{T}, \ldots,\left(u^{p j}\right)^{T}\right) \in R_{+}^{Q}$. Further we denote $u \in\left(R_{+}^{Q}\right)^{q}$ by $u=\left(u(1)^{T}\right.$, $\left.u(2)^{T}, \ldots, u(j)^{T}, \ldots, u(q)^{T}\right)$. Let $G \in C\left[R_{+} \times\left(R_{+}^{Q}\right)^{q} \times\left(\mathscr{C}_{+}^{Q}\right)^{q},\left(R_{+}^{Q}\right)^{q}\right]$, where $Q=\prod_{i=1}^{p} q_{i}$. The function $G(t, u, \sigma)$ is said to possess a block quasimonotone nondecreasing property in $u$ for each $(t, \sigma)$ if for each $j \in I(1, q)$, (a) the block $G_{j}(t, u, \sigma)$ is nondecreasing in 
$u(l)$ for $l \neq j$, and (b) for $l=j$ and each $i \in I(1, p), G^{i j}(t, u, \sigma)$ is quasimonotone nondecreasing in $u^{i j}$ and nondecreasing in $u^{k j}$ for $k \neq i$ and $k \in I(1, p)$.

Lemma 2.4. Assume that

(i) $g^{i j} \in C\left[R_{+} \times R_{+}^{q_{i}} \times \mathscr{C}_{+}^{q_{i}}, R_{+}^{q_{i}}\right], g^{i j}\left(t, u^{i j}, \sigma^{i j}\right)$ is concave in $\left(u^{i j}, \sigma^{i j}\right)$ for each $t$, quasimonotone nondecreasing in $u^{i j}$ for each $\left(t, \sigma^{i j}\right) \in R_{+} \times \mathscr{C}_{+}^{q_{i}}$, and increasing in $\sigma^{i j}$ for each $\left(t, u^{i j}\right) \in R_{+} \times R_{+}^{q_{i}}$, for each $i \in I(1, p)$, and $j \in I(1, q)$;

(ii) $h^{i j} \in C\left[R_{+} \times\left(R_{+}^{Q}\right)^{q} \times\left(\mathscr{C}_{+}^{Q}\right)^{q}, R_{+}^{q_{i}}\right], h^{i j}(t, u, \sigma)$ is concave in $(u, \sigma)$ for each $t$, increasing in $u(l)$ for $l \neq j$, for $\ell=j$ quasimonotone nondecreasing in $u^{i j}$, and nondecreasing $u^{k j}$ for $k \neq i$ for each $(t, \sigma) \in R_{+} \times \mathscr{C}_{+}^{Q}$ any $k \in I(1, p)$, and increasing in $\sigma$ for each $(t, u) \in\left(R_{+} \times R_{+}^{Q}\right)^{q}$ for each $j \in I(1, q)$;

(iii) $G \in C\left[R_{+} \times\left(R_{+}^{Q}\right)^{q} \times\left(\mathscr{C}_{+}^{Q}\right)^{q},\left(R_{+}^{Q}\right)^{q}\right]$, and $G(t, u, \sigma)$ is defined by

$$
G(t, u, \sigma)=\left(G^{1}(t, u, \sigma)^{T}, \ldots, G^{j}(t, u, \sigma)^{T}, \ldots, G^{q}(t, u, \sigma)^{T}\right)^{T}
$$

where, $j \in I(1, q)$ and $G^{j} \in C\left[R_{+} \times\left(R_{+}^{Q}\right)^{q} \times\left(\mathscr{C}_{+}^{Q}\right)^{q},\left(R_{+}^{Q}\right)\right]$, and

$$
\begin{aligned}
& G^{j}(t, u, \sigma)=\left(G^{1 j}(t, u, \sigma)^{T}, \ldots, G^{i j}(t, u, \sigma)^{T}, \ldots, G^{p j}(t, u, \sigma)^{T}\right)^{T}, \\
& G^{i j}(t, u, \sigma)=g^{i j}\left(t, u^{i j}, \sigma^{i j}\right)-\pi_{j j} u^{i j}+h^{i j}(t, u(j), \sigma(j))+\sum_{l \neq j}^{q} \pi_{j l} u^{l j},
\end{aligned}
$$

Then

where $i \neq k, \pi_{j l} \geq 0$ for $j, l \in I(1, q)$, and $i, k \in I(1, p)$.

$$
\frac{d}{d t} u=G(t, u, \sigma), \quad u_{t_{0}}=\sigma_{0}
$$

has a maximal solution for $t \geq t_{0}$.

Proof. We note that $G$ defined in (2.4) satisfies the block quasimonotone nondecreasing property in $u$. The rest of the proof of the lemma can be constructed by following the argument used in the proofs of the results in [15-17]. The details are left to the reader.

Now, we are ready to present a result that is similar to [9, Theorem 3.1].

Theorem 2.5. Assume that

(a) $g^{i j}, h^{i j}, G^{i j}$, and $G$ satisfy the conditions of Lemma 2.4;

(b) $r(t)=r\left(t_{0}, \sigma_{0}\right)(t)$ is the maximal solution of system of comparison differential equations (2.6) existing for $t \geq t_{0}$;

(c) $z^{i}\left(t, s, z_{0}^{i}\right)$ solution process of auxiliary system (DAS) in [9] through $\left(s, \varphi^{i}(0)\right), t_{0} \leq$ $s \leq t$ and its second derivative $\left(\partial^{2} / \partial x^{i} \partial x^{i}\right) z^{i}\left(t, s, z_{0}^{i}\right)$ is locally Lipschitzian in $z_{0}^{i}$ for each $i \in I(1, p)$; 
(d) $V^{i} \in C\left[(-\tau, \infty) \times R^{n_{i}} \times R, R_{+}^{q_{i}}\right]$ and $(\partial / \partial t) V^{i}\left(s, z^{i}, y\right),(\partial / \partial x) V^{i}\left(s, z^{i}, y\right)$, and $\left(\partial^{2} / \partial x^{2}\right) V\left(s, z^{i}, y\right)$ exist and are continuous on $[-\tau, \infty) \times R^{n_{i}} \times R ;\left(\partial^{2} / \partial x^{2}\right) V^{i}\left(s, z^{i}\right.$, $y)$ is locally Lipschitzian in $z^{i}$ for $t_{0} \leq s \leq t$, and $L_{i}^{D} V^{i}\left(s, \varphi^{i}, z^{i}\left(t, s, \varphi^{i}(0)\right), \eta(s)\right)$ satisfies the following relation:

$$
L_{i}^{D} V^{i}\left(s, \varphi^{i}, z^{i}\left(t, s, \varphi^{i}(0)\right), j\right) \leq g^{i j}\left(s, V^{i}\left(s, z^{i}\left(t, s, \varphi^{i}(0)\right), j\right), V_{s}^{i j}\right)
$$

for each $i \in I(1, p)$ and $j \in I(1, q)$;

(e) for each $i \in I(1, p)$ and $j \in I(1, q)$ the interaction functions among the subsystems satisfy the inequality

$$
I^{i}\left(\mathbf{c}^{i}(t, s, \varphi, j)\right) \leq h^{i j}\left(s, V(s, z(t, s, \varphi(0)), j), V_{s}^{j}\right)
$$

where, $I^{i}$ is as defined in (2.2) and

$$
\begin{aligned}
& V(s, z(t, s, \varphi(0)), j) \\
& \quad=\left(V^{1}\left(s, z^{1}\left(t, s, \varphi^{1}(0)\right), j\right)^{T}, \ldots, V^{p}\left(s, z^{p}\left(t, s, \varphi^{p}(0)\right), j\right)^{T}\right)^{T}
\end{aligned}
$$

(f) $x\left(t_{0}, \phi_{0}\right)(t)=x(t)$ is a solution process of large-scale system (LHS) through $\left(t_{0}, \phi_{0}\right)$ and $E[V(s, z(t, s, x(s)), \eta(s))]$ exists for $t_{0} \leq s \leq t, i \in I(1, p)$, and

$$
E\left[V\left(t_{0}+\theta, z(t, s+\theta, \varphi(\theta)), \eta\left(t_{0}\right)\right) \mid \eta\left(t_{0}\right)=j_{0}\right] \leq \sigma_{0}^{i j_{0}}(\theta), \quad \text { for all } \theta \in[-\tau, 0] .
$$

Then

$$
E\left[V\left(t, x\left(t_{0}, \phi_{0}\right)(t), j\right) \mid x_{t_{0}}=\varphi_{0}\right] \leq r^{j}\left(t_{0}, \sigma_{0}\right)(t), \quad t \geq t_{0}
$$

for all $t \geq t_{0}, j \in I(1, q)$.

Proof. From (2.2), assumptions (c), (d), and (e), and employing the standard argument, we have

$$
\begin{aligned}
& L_{i}^{C} V^{i}\left(s, \varphi, z^{i}\left(t, s, \varphi^{i}(0)\right), \eta(s)\right) \\
& \quad=L_{i}^{D} V^{i}\left(s, \varphi^{i}, z^{i}\left(t, s, \varphi^{i}(0)\right), \eta(s)\right)+I^{i}\left(\mathbf{c}^{i}(t, s, \varphi, \eta(s))\right) \\
& \quad \leq g^{i j}\left(s, V^{i}\left(s, z^{i}\left(t, s, \varphi^{i}(0)\right), j\right), V_{s}^{i j}\right)+h^{i j}\left(s, V(s, z(t, s, \varphi(0)), j), V_{s}^{j}\right),
\end{aligned}
$$

for all $i \in I(1, p)$ and $j \in I(1, q)$. Let $x\left(t_{0}, \varphi_{0}\right)(t)=x(t)$ be a solution process of largescale system (LHS) through $\left(t_{0}, \phi_{0}\right)$. By following the argument used in the proof of $[9$, Theorem 3.1] and using (2.3), we arrive at

$$
D^{+} m^{i j}(s) \leq L_{i}^{C} V^{i}\left(s, \varphi, z^{i}\left(t, s, \varphi^{i}(0)\right), j\right)+D_{\eta}^{+} V^{i}\left(s, z^{i}\left(t, s, \varphi^{i}(0)\right), j\right) .
$$


6 Large-scale stochastic systems. Part III. Qualitative analysis

From [9, Theorem 3.1, in particular, (3.3), (3.11), and (3.12)], (2.12), (2.13), assumption (a), and notations in Definition 2.3, we have

$$
D^{+} m(s) \leq G\left(s, m(s), m_{s}\right), \quad \text { for } t_{0} \leq s \leq t,
$$

and from (2.10), we have

$$
m(\theta) \leq \sigma_{0}(\theta), \quad \theta \in[-\tau, 0] .
$$

Now by following the standard argument $[1,3,4,8,15-17]$, the proof of the theorem can be completed.

Remark 2.6. A corollary similar to [9, Corollary 3.1] can be formulated, analogously.

Remark 2.7. If $f^{i} \equiv 0$, then Theorem 2.5 reduces to the usual comparison theorems for studying large-scale systems [2, 3, 6, 8, 14-17] as special cases.

Remark 2.8. By following arguments used in [9, Examples 4.1 and 4.2], one can illustrate the scope of Theorem 2.5. Details are left to the reader.

\section{Qualitative analysis of large-scale systems}

Now, we are ready to formulate the convergence and stability results for stochastic largescale hereditary system (LHS).

First, we present very general results regarding convergence and stability properties of the solution process of (LHS).

Theorem 3.1. Assume that all the hypotheses of Theorem 2.5 are satisfied. Further assume that

(i) for each $t \in R_{+}, V^{i}(t, x, j)$ satisfies the inequality

$$
a\left(\|x\|^{\gamma}\right) \leq \sum_{j=1}^{q} \sum_{i=1}^{p} \sum_{k=1}^{q_{i}} V_{k}^{i}(t, x, j) \leq b\left(\|x\|^{\gamma}\right),
$$

where $a \in \mathscr{V} \mathscr{Y}$ and $b \in \mathscr{C} \mathscr{Y}$;

(ii) the maximal solution process $r\left(t_{0}, \sigma_{0}\right)(t)$ of (2.6) converges to zero.

Then, the solution process $x\left(t_{0}, \varphi_{0}\right)(t)$ of (LHS) converges in the $\gamma$ th mean to the zero.

In the following, we provide the sufficient conditions to insure the almost-sure convergence of a solution process of (LHS) to the zero vector.

Theorem 3.2. Assume that hypotheses of Theorem 2.5 are satisfied. Further assume that for each $i \in I(1, p)$ and $j \in I(1, q)$ :

(i) the function $V^{i}\left(t, x^{i}, y\right)$ is such that $V^{i}(t, 0, y) \equiv \theta$ is the unique minimum of $V^{i}(t$, $\left.x^{i}, y\right)$, where $\theta \in R_{+}^{q_{i}}$ denotes the zero vector; 
(ii) for each $j \in I(1, q)$, there exists a block diagonal matrix function $\Gamma^{j}(t)$ such that $\Gamma^{j} \in \mathscr{C}\left[\mathscr{R}_{+}, R_{+}^{Q} \times R_{+}^{Q}\right]$, and it is defined by

$$
\begin{gathered}
\Gamma^{j}(t)=\operatorname{diag}\left\{T^{1 j}(t), T^{2 j}(t), \ldots, T^{i j}(t), \ldots, T^{p j}(t)\right\}, \\
T^{i j}(t)=\operatorname{diag}\left\{\kappa_{11}^{i j}(t), \kappa_{22}^{i j}(t), \ldots, \kappa_{q_{t} q_{t}}^{i j}(t)\right\}_{q_{i} \times q_{i}},
\end{gathered}
$$

where $\kappa_{k k}^{i j}(t)>0$ for $1 \leq k \leq q_{i}$ and $1 \leq i \leq p$, and for $\sigma(j)(0), \psi(j)(0) \in R_{+}^{Q}$, $\sigma(j)(0) \geq \psi(j)(0)$

$$
G^{j}(t, \sigma(j)(0), \sigma)-G^{j}(t, \psi(j)(0), \psi) \geq-\Gamma^{j}(t)(\sigma(j)(0)-\psi(j)(0)) ;
$$

(iii) $\int_{0}^{\infty}\left[G\left(t, E[r(t)], E\left[r_{t}\right]\right)+\Gamma(t) E[r(t)]\right] d t<\infty$, and

$$
G(t, 0,0) \geq 0, \quad \text { for } t \geq t_{0},
$$

where $r(t)=r\left(t_{0}, \sigma_{0}\right)(t)$ is the maximal solution of (2.6) through $\left(t_{0}, \sigma_{0}\right)$ and $\Gamma(t)=$ $\operatorname{diag}\left\{\Gamma^{1}(t), \Gamma^{2}(t), \ldots, \Gamma^{q}(t)\right\}_{q \times q} ;$

(iv) the mean of the maximal solution $E[r(t)]=E\left[r\left(t_{0}, \sigma_{0}\right)(t)\right]$ of $(2.6)$ through $\left(t_{0}, \sigma_{0}\right)$ converges to the zero vector as $t \rightarrow \infty$.

Then a solution process of (LHS) converges to the zero vector (a.s.) as $t \rightarrow \infty$.

Proof. The proof of the theorem can be constructed by following the argument of [10, Theorem 3.2], and the argument used in the proofs of the results in $[1,3,8,13,14]$. The details are left to the reader.

Now, we present sufficient conditions that assure the almost-sure stability properties of the trivial solution process of (LHS).

Theorem 3.3. Assume that hypotheses of Theorem 2.5 are satisfied. Further assume that

(i) for each $t \in R_{+}, V^{i}(t, x, j)$ satisfies the inequality

$$
a\left(\|x\|^{\gamma}\right) \leq \sum_{j=1}^{q} \sum_{i=1}^{p} \sum_{k=1}^{q_{i}} V_{k}^{i}(t, x, j) \leq b\left(\|x\|^{\gamma}\right),
$$

where $a \in \mathscr{V} \mathscr{Y}$ and $b \in \mathscr{C} \mathscr{K}$;

(ii) $\mathbf{F}(t, 0, \eta(t)) \equiv 0$ and $G(t, 0,0) \equiv 0$. Then,

(a) the stability of the trivial solution of $r(t) \equiv 0$ of (2.6) implies the $\gamma$ th mean stability of the trivial solution process $x(t) \equiv 0$ of (LHS);

(b) the asymptotic stability of the trivial solution of $r(t) \equiv 0$ of (2.6) implies the $y$ th mean asymptotic stability of the trivial solution process $x(t) \equiv 0$ of (LHS).

Proof. The proof of the theorem can be formulated by following the argument used in the proof of [10, Theorem 3.3]. The details are left to the reader.

We present sufficient conditions that assure the almost-sure stability properties of the trivial solution process of (LHS). 
8 Large-scale stochastic systems. Part III. Qualitative analysis

Theorem 3.4. Assume that hypotheses of Theorem 2.5 are satisfied. Further assume that for each $i \in I(1, p)$ and $j \in I(1, q)$ :

(i) for each $t \in R_{+}, V^{i}(t, x, j)$ satisfies the inequality

$$
a\left(\left\|x^{i}\right\|^{\gamma}\right) \leq \sum_{j=1}^{q} \sum_{i=1}^{p} \sum_{k=1}^{q_{i}} V_{k}^{i}(t, x, j) \leq b\left(\left\|x^{i}\right\|^{\gamma}\right),
$$

where $a \in \mathscr{V} \mathscr{Y}$ and $b \in \mathscr{C} \mathscr{Y}$;

(ii) for each $j \in I(1, q)$, there exists a block diagonal matrix function $\Gamma^{j}(t)$ such that $\Gamma^{j} \in \mathscr{C}\left[\mathscr{R}_{+}, R_{+}^{Q} \times R^{Q}\right]$, and it is defined by

$$
\begin{gathered}
\Gamma^{j}(t)=\operatorname{diag}\left\{T^{1 j}(t), T^{2 j}(t), \ldots, T^{i j}(t), \ldots, T^{p j}(t)\right\}, \\
T^{i j}(t)=\operatorname{diag}\left\{\kappa_{11}^{i j}(t), \kappa_{22}^{i j}(t), \ldots, \kappa_{q_{t} q_{t}}^{i j}(t)\right\}_{q_{i} \times q_{i}},
\end{gathered}
$$

where $\kappa_{k k}^{i j}(t)>0$ for $1 \leq k \leq q_{i}$ and $1 \leq i \leq p$, and for $\sigma(j)(0), \psi(j)(0) \in R_{+}^{Q}$, $\sigma(j)(0) \geq \psi(j)(0)$,

$$
G^{j}(t, \sigma(j)(0), \sigma)-G^{j}(t, \psi(j)(0), \psi) \geq-\Gamma^{j}(t)(\sigma(j)(0)-\psi(j)(0)) ;
$$

(iii) $\int_{t_{0}}^{\infty}\left[G\left(t, E[r(t)], E\left[r_{t}\right]\right)+\Gamma(t) E[r(t)]\right] d t<\infty$, where $r(t)=r\left(t_{0}, \sigma_{0}\right)(t)$ is the maximal solution of (2.6) through $\left(t_{0}, \sigma_{0}\right)$ and $\Gamma(t)=\operatorname{diag}\left\{\Gamma^{1}(t), \Gamma^{2}(t), \ldots, \Gamma^{q}(t)\right\}_{q \times q}$; Then,

(iv) $\mathbf{a}^{i}(t, 0, \eta(t)) \equiv 0, \mathbf{c}^{i}(t, 0, \eta(t)) \equiv 0$ and $G(t, 0,0) \equiv 0$.

(a) the stability of the trivial solution of $r(t) \equiv 0$ of (2.6) implies the almost-sure stability of the trivial solution process $x(t) \equiv 0$ of (LHS);

(b) the asymptotic stability of the trivial solution of $r(t) \equiv 0$ of (2.6) implies the $\gamma$ th mean asymptotic stability of the trivial solution process $x(t) \equiv 0$ of (LHS).

Proof. The proof of the theorem can be constructed by employing the proof of [10, Theorem 3.4]. The details are left to the reader.

Remark 3.5. Examples similar to [10, Examples 4.1 and 4.2] can be constructed. Further, additional examples can be worked out as in the theory of large-scale systems $[3,7,8]$.

\section{Conclusions}

From our stability analysis, we can draw several conclusions. We briefly state some of them. However, details will appear elsewhere. Stability conditions in [10], for example:

$$
\begin{array}{r}
\pi_{j j}-\alpha^{i j}(t)-\tau \beta^{i j}(t)>0, \quad j \in I(1, q), \\
\pi_{j j}-\alpha^{i j}(t)-\tau \beta^{i j}(t)-d_{j}^{-1} \sum_{l \neq j}^{q} d_{l} \pi_{l j}>\alpha>0,
\end{array}
$$

for some $\alpha>0$ for each $i \in I(1, q)$. 
(1) These conditions imply that $q \times q$ matrices:

$$
A(t, \tau, \pi)=\left(a_{j l}(t, \tau, \pi)\right)_{q \times q}, \quad a_{j l}(t, \tau, \pi)= \begin{cases}-\pi_{j j}+\alpha^{i j}(t)+\tau \beta^{i j}(t), & l=j, \\ \pi_{j l}, & l \neq j,\end{cases}
$$

are Metzler and stable matrices [18].

(2) From condition (4.2), we conclude that the weighted self-inhibitory nonhereditary and structural perturbation effects are larger than the cross-coupling structural perturbation effects $[2,6-8,11,18]$.

(3) These conditions are algebraically simple and easy to compute.

(4) They are expressed in terms of rate coefficients, time-delay, and intensity matrix.

(5) Condition (4.2) gives an estimate on time-delay $\tau$, magnitude of intensity matrix, and magnitude of hereditary interactions.

(6) They reflect the hereditary effects characterized by $\tau$ as destabilizing agents $[2,6-$ $8,11]$.

(7) They are sufficient, however, they are reliable $[2,6-8,13,14,18]$.

(8) They are robust with respect to parametric changes of the system $[2,6-8,13,14$, $18]$.

(9) They show that structural perturbations can be considered as stabilizing agents.

(10) They shed a light on a fundamental problem on fundamental issues, namely: (i) "complexity versus stability," (ii) "stochastic versus deterministic," and (iii) "hereditary versus nonhereditary" in nonlinear nonstationary dynamic processes in biological, physical, and social sciences $[2,3,6,11,14,18]$.

\section{References}

[1] G. S. Ladde, Systems of functional differential inequalities and functional differential systems, Pacific Journal of Mathematics 66 (1976), no. 1, 161-171.

[2] Competitive processes. I. Stability of hereditary systems, Nonlinear Analysis. Theory, Methods \& Applications 1 (1977), no. 6, 607-631.

[3] _ Stability of general systems in biological, physical and social sciences, Applied General Systems Research (Internat. Conf., State University of New York, Binghamton, NY, 1977) (G. J. Klir, ed.), NATO Conf., Ser.: Systems Sci., vol. 5, Plenum, New York, 1978, pp. 575-587.

[4] Systems of differential inequalities and stochastic differential equations. IV, Journal of Mathematical Physics 19 (1978), no. 8, 1733-1741.

[5] , Time lag versus stability, IEEE Transactions on Automatic Control 23 (1978), no. 1, $84-85$.

[6] __ Stochastic stability analysis of model ecosystems with time-delay, Differential Equations and Applications in Ecology, Epidemics, and Population Problems (Claremont, Calif, 1981) (S. N. Busenberg and K. L. Cooke, eds.), Academic Press, New York, 1981, pp. 215-228.

[7] St_ Stochastic delay differential systems, Proceedings of the International Symposium on Functional Differential Equations, Kyoto, Japan, World Scientific, New Jersey, 1991, pp. 204212.

[8] Stabilizing and oscillating hereditary and random structural perturbations effects on multispecies processes, Proceedings of Conference on: Nonlinear Systems: Modelling, Simulation and Applications (S. B. Agase, ed.), Science College, Nanded, 2003, pp. 1-20. 
10 Large-scale stochastic systems. Part III. Qualitative analysis

[9] L Large-scale stochastic hereditary systems under Markovian structural perturbations I: variational comparison theorems, Journal of Applied Mathematics and Stochastic Analysis 2006 (2006), Article ID 19871, 1-11.

[10] _ Large-scale stochastic hereditary systems under Markovian structural perturbations II: qualitative analysis of isolated subsystems, Journal of Applied Mathematics and Stochastic Analysis 2006 (2006), Article ID 67268, 1-14.

[11] _ Qualitative Analysis of Competitive-Cooperative Process in Biological, Physical and Social Sciences, Monograph in Preparation.

[12] G. S. Ladde and V. Lakshmikantham, Competitive-cooperative processes and stability of diffusion systems, Applied Stochastic Processes (Proceedings of Conf., University of Georgia, Athens, Ga, 1978) (G. Adomian, ed.), Academic Press, New York, 1980, pp. 83-108.

[13] G. S. Ladde and B. A. Lawrence, Stability and convergence of large-scale stochastic approximation procedures under Markovian structural perturbations, Differential Equations and Dynamical (D. Bahuguna, ed.), Narosa, New Delhi, 2004, pp. 25-48.

[14] G. S. Ladde and D. D. Šiljak, Connective stability of large-scale stochastic systems, International Journal of Systems Science 6 (1975), no. 8, 713-721.

[15] V. Lakshmikantham and S. Leela, Differential and Integral Inequalities: Theory and Applications. Vol. I: Ordinary Differential Equations, Academic Press, New York, 1969.

[16] _ Differential and Integral Inequalities: Theory and Applications. Vol. II: Functional, Partial, Abstract, and Complex Differential Equations, Academic Press, New York, 1969.

[17] G. R. Shendge, Finite systems of functional differential inequalities and minimax solutions, Acta Mathematica Academiae Scientiarum Hungaricae 25 (1974), no. 1-2, 21-30.

[18] D. D. Šiljak, Large-Scale Dynamic Systems. Stability and Structure, North-Holland, New York, 1978.

G. S. Ladde: Department of Mathematics, The University of Texas at Arlington, Arlington,

TX 76019, USA

E-mail address: ladde@uta.edu 


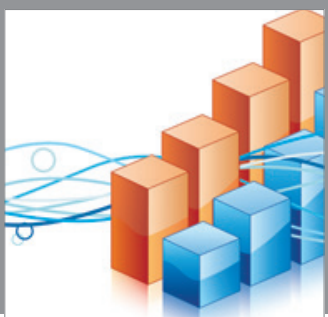

Advances in

Operations Research

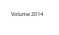

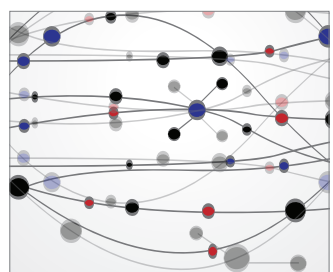

\section{The Scientific} World Journal
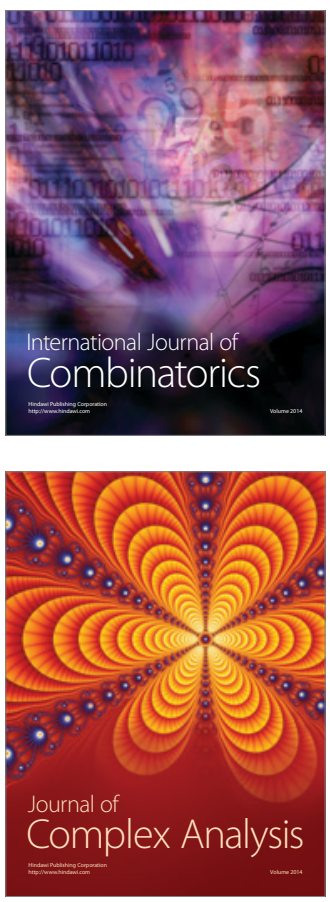

International Journal of

Mathematics and

Mathematical

Sciences
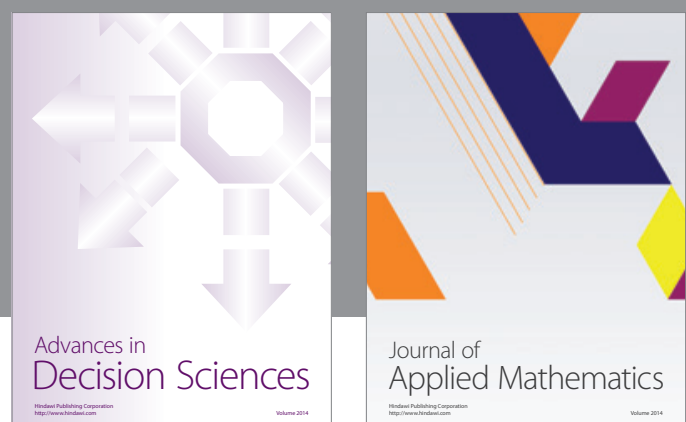

Journal of

Applied Mathematics
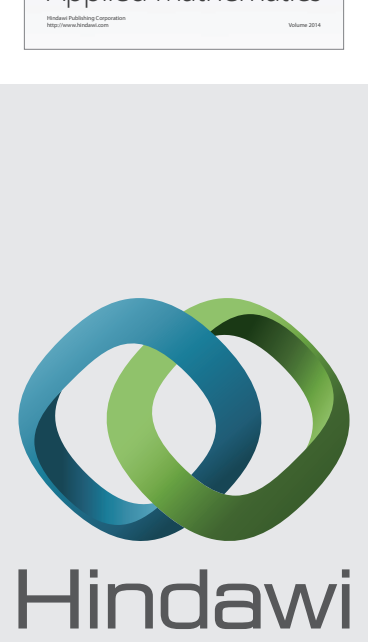

Submit your manuscripts at http://www.hindawi.com
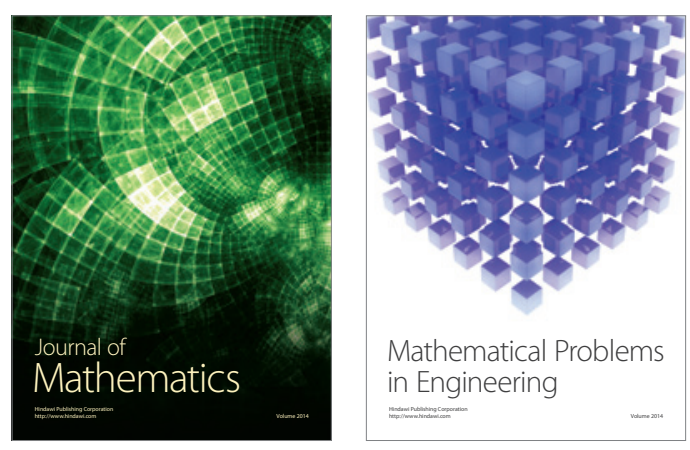

Mathematical Problems in Engineering
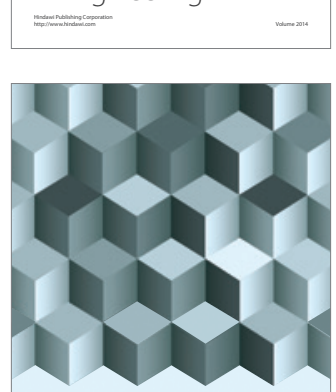

Journal of

Function Spaces
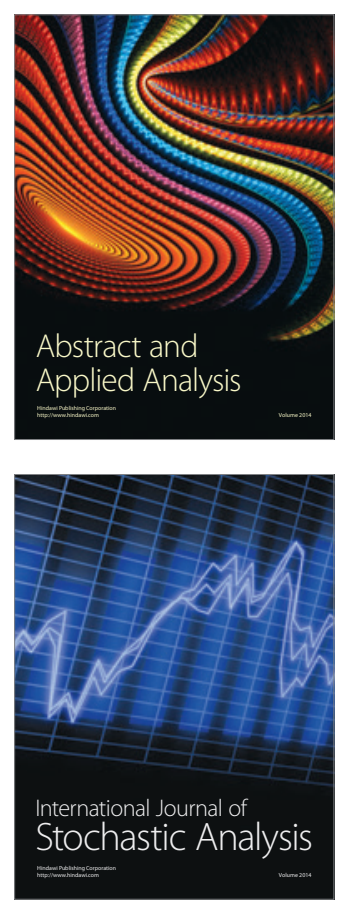

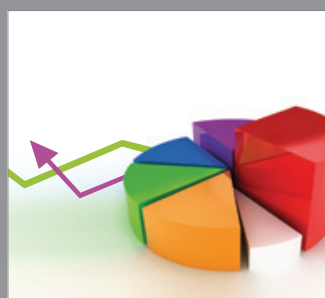

ournal of

Probability and Statistics

Promensencen
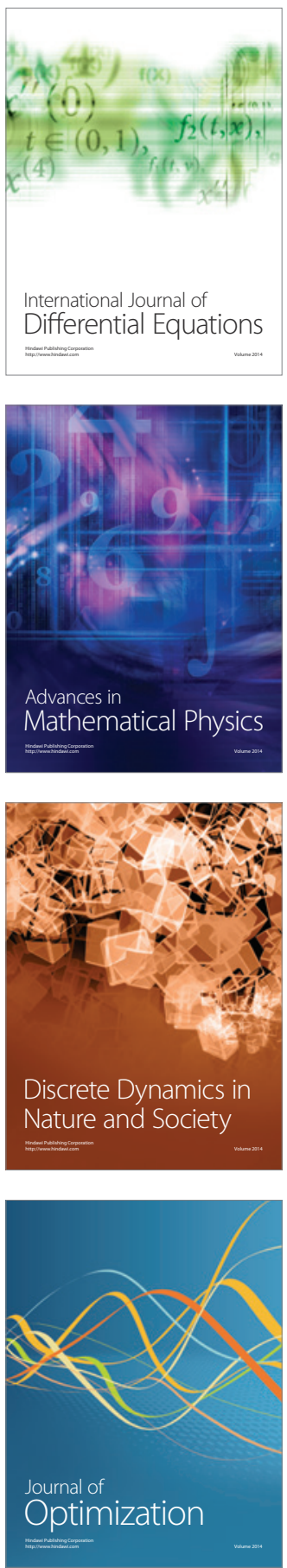